\title{
Modified algorithm research of target tracking of mean shift infrared imagery
}

\author{
YUE Ming-kai \\ Beijing Institute of Technology, Beijing, China \\ Shenyang Ligong University, Shenyang, Liaoning, China \\ e-mail:13032486996@163.com
}

\author{
DENG Jia-hao \\ Beijing Institute of Technology, Beijing, China \\ e-mail: bitdjh@sohu.com
}

\begin{abstract}
The moving target tracking of infrared imagery has been usually applied in the field of imaging and guidance military reconnaissance of weapon, and intelligent transportation system, video surveillance, etc. for civil use. The application of mean shift in the tracking of infrared target was investigated and the target model was built by utilizing the weighted kernel histogram of grayscale likelihood ratio, and then the disadvantages of mean shift were analyzed and modified in the target tracking. The modified algorithm has better tracking effect by contrasting experiments than the conventional one.
\end{abstract}

Keywords-Infrared imagery target; Mean shift; Weighted kernel histogram;Target tracking

\section{INTRODUCTION}

The mean shift has some advantages and disadvantages when it is applied in the field of infrared target tracking as the infrared imagery itself has some imaging characteristics. The target main characteristic is shown by color, actually by the similar matching of color and texture, in the visible image, so the target model with only grayscale information would be subjected to background interference by similar grayscale information, which easily causes the offset of tracking results and target actual position. The window size keeps unchangeable when the classic mean shift is tracking. However, the target size is impossibly unchangeable as the target path may be from faraway to nearby or vice versa and the change may cause the tracking failure. Aiming at the above problem that the mean shift can't change the window width of kernal, the mean shift will be modified to solve it.

\section{MODIFICATION OF TARGET MODELING}

The infrared imaging quality is lower as the information appeared in image , the edge and texture is a little, as well as low signal to noise ratio. The poor distinction between the target and the background will cause the target selection difficult in the complex condition. The methode of grayscale likelihood ratio weighted function will reduce the effection of the complex background in some extent when modeling the target. The methode of grayscale likelihood ratio weighted kernal histogram will be utilized to solve the insufficient correction of the target complex background, as well as modeling of infrared target in luminance to distance space.

\section{A. Modeling of infrared target in luminance to distance space}

The characteristic of target region exactly extracted and expressed is a key problem before the tracking of infrared target. The tracking of target is that the intrested infrared target could be separated from the complex background and continually positioned. Generally, we decide if the region is the target by computing the similarity of grayscale between the infrared target region and the template one. As for the rigid target, the above methode can represent the target characteristic. However, as for the nonrigid target, such as people and animals, this kind of targets are completely elastic and random, so the representation would always lose or misjust the target characteristics. Actually, the luminance characteristic of infrared image in the target region has some compression and the scale factor has a major interruption on the extraction of inertia charateristic, the classification capacity by using the above methodes isn't good in the course of tracking. Therefore,we have modified the above representation model of the target and the luminance to distance joint histogram characteristic methode of better representation of nonrigid target model has been constructed. Certainly, the modified methode has the same better effect in the tracking of rigid target.

Suppose that the target region of an infrared image can expressed as a rectangle of length $m$ and width $n$, then the tracking problem is the characteristic analysis of distance and grayscale in the rectangle region. $I(x, y)$ denoted the grayscale of some pixel, then the construction methode of luminance to distance 2D histogram can expressed as follows:

\section{(1) Luminance characteristic histogram}

Construct a function to make luminance (grayscale) of every pixel in an infrared image to map the corresponding series of the histogram, then the luminance histogram, which could represent the luminance of the target region, would be obtained. 


$$
\begin{gathered}
H_{1}(f)=\sum_{i=1}^{n} \sum_{j=1}^{m} \delta\left[I^{\prime}(x, y)-f\right] \\
I^{\prime}(x, y)=\left\{\begin{array}{lr}
1 & I(x, y)=0 \\
\text { floor }\left(\frac{N_{1} I(x, y)}{255}\right) & \text { 其他 }
\end{array}\right.
\end{gathered}
$$

Where $f=1,2 \ldots, \quad N_{1}$ is the series of the histogram, $\delta$ is the delta function.

\section{(2) Distance characteristic histogram}

At first, compute the distance from every pixel to the center of the image in the recangle region:

$$
d(x, y)=\sqrt{\left(x-x_{0}\right)^{2}+\left(y-y_{0}\right)^{2}}
$$

Where the point $\left(x_{0}, y_{0}\right)$ denotes the center point of the rectangle of the tracking target.

Then map the distances of every pixel and center to the corresponding serier of the histogram, then the distance histogram will be obtained:

$$
\begin{gathered}
H_{2}(k)=\sum_{i=1}^{n} \sum_{j=1}^{m} \delta\left[d^{\prime}(x, y)-k\right] \\
d^{\prime}(x, y)=\left\{\begin{array}{l}
1 \quad d(x, y)=0 \\
f l o o r\left(\frac{2 N_{2} d(x, y)}{\sqrt{m^{2}+n^{2}}}\right)
\end{array} \quad\right. \text { 其他 }
\end{gathered}
$$

Where $k=1,2 \ldots, N_{2}$ is the histogram series, $\delta$ is the delta function.

\section{(3) Joint histogram construction}

The luminance to distance joint histogram will be obtained when mixing the above luminance characteristic histogram with the distance one by the means of the following methode.

$$
H(k, f)=\sum_{x=1}^{n} \sum_{y=1}^{m} \delta\left[d^{\prime}(x, y-k)\right] \delta\left[I^{\prime}(x, y)-f\right]
$$

Where, at first, reduce the dimensionality of the 2D histogram of series $N_{1}, N_{2}$ and then change into 1D joint histogram of series $N_{1} \times N_{2}$. After that, normalization processing is carried out. At last, the suitable histogram series will be found for optimized tracking after the quantative analysis is implemented according to the classification results.

\section{B. Infrared target representation model of grayscale likelihood ratio weighted kernal histogram.}

In order to increase the classification capacity between the target and the background in the infrared image, the method of grayscale likelihood ratio weighted kernal histogram is very well to represent the infrared target model.
Combining the infrared target model with the kernal histogram increases the contribution of the grayscale constitution of target characteristics, therefore, the representation capacity increases, which is more beneficial to track the target precisely.

It is usual to choose the rectangle region in the course of target tracking, then find a little bigger concentric rectangle region as the background. Generally, we select a new rectangle, the length and width of which is 1.5 or 2 times that the original rectangle, and then build the target and the background histogram model. After that, turn into the discrete probability density. The discrete probability density of the target model denotes $h_{\text {tar }}(i)$ and the one of the background $h_{b g}(i)$, then the grayscale likelihood ratio can be defined as follows:

$$
L(i)=\log \frac{\max \left\{h_{t a r}(i), \delta\right\}}{\max \left\{h_{b g}(i), \delta\right\}}
$$

Where $\delta$ has a very small value and generally selected as 0.001 . The function of this value is in case that the denominator is zero or $L(i)$ is zero. Therefore the grayscale likelihood weighted background histogram is:

$$
\hat{q}_{u}=C L(u) \sum_{i=1}^{n} k\left(\left\|\frac{x_{i}-x_{0}}{h}\right\|^{2}\right) \delta\left[b\left(x_{i}\right)-u\right]
$$

Where $C$ represents a normalization coefficient and $L(u)$ is a grayscale likelihood ratio function with $x_{0}$ as the center position. By using the same methode, the grayscale likelihood ratio weighted background histogram for the pixels of the candidate target region can expressed as follows:

$$
\hat{p}_{u}=C L(u) \sum_{i=1}^{n_{k}} k\left(\left\|\frac{y-x_{i}}{h}\right\|^{2}\right) \delta\left[b\left(x_{i}\right)-u\right]
$$

Where $L(u)$ is a grayscale likelihood ratio function with $y$ as the center position.

The small rectangle region in Fig. 1 is the target one in a more complex infrared image. It can be seen from the Fig.1 that the distinction of the target and the background isn't obvious, and they have a lot of similarity and their pixels have a strong interruption. The almost same pixel values of the target and the background have a severe influence on the correction of mapping the target model to the candidate one in the complex infrared target image, which makes the mean shift converge to the wrong position.

As can be seen from Fig.1, the small rectangle region is the pixels of the target whilst the region between the big rectangle and the small rectangle is the pixels of the background. In order to reduce the influence on the pixels of the background to the target or the candidate models, generally the partial pixels nearby the target are selected to compute the grayscale likelihood ratio. 


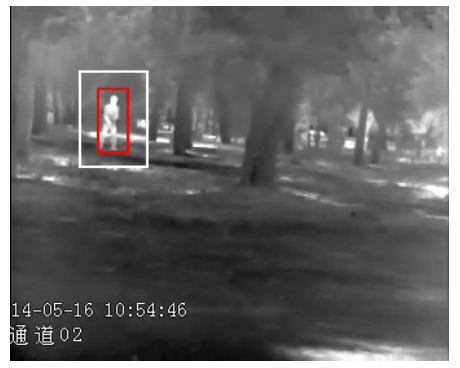

Fig.1. Gray histogram likelihood ratio weighted nuclear infrared target characterization.

\section{Tracking algorithm of self-adapting update of kernel window width.}

The target size will change in some extent as its moving path may be from faraway to nearby or vice versa in practical application. The threshold segmentation for the tracked infrared target is carried out in this papar and then the segmented area is computed. After that, it will update according to the average value of practical target region in a newer period. The tendency of the infrared target is determined by the change of the average region in a period whilst the level of the target by the one of the average region.

Due to the imaging quality of the infrared image itself, especially for the non-rigid target, such as human, the change of the human target posture also makes the segmented region change greatly even if the human target don't change from faraway to nearby or vice versa. In order to reduce the interruption of the outer factors, the average value of the segmented region was utilized in this paper. Generally, the consecutive frame images change little, so it is unnecessary to update the kernel window width every time when tracking, which will reduce the calculated amount greatly. The updated period $\mathrm{T}$ would set as 15 frames in this paper through many experiments, but this value could reduce suitably based on the practical experiments and application conditions.

\section{RESULTS AND ANALYSIS}

At first, the contrast experiment were carried out by selecting two groups of real shot infrared image sequence and all the experiments were realized with the Matlab (R2010) based on PC of i5,2.7Hz,2G internal storage. The size of the image is $352 \times 288$. Both group also were composed of group $\mathrm{A}$ and $\mathrm{B}$ to contrast. The image sequence of group $\mathrm{A}$ was the infrared target tracking images by using the conventional mean shift while the one of group B by using the modified kernel window width self-adapting update method, which can test the tracking effect of modified algorithm.

Fig. 2 is a group of infrared image sequence from faraway to nearby. The target in the image is the human body and it zoomed in gradually during the tracking procedure. The infrared image sequence of group A used conventional mean shift to track the target whilst the ones of group B used the algorithm of kernel window width self-adapting update.

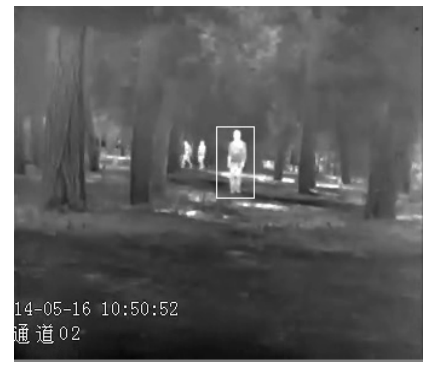

the 1st frame

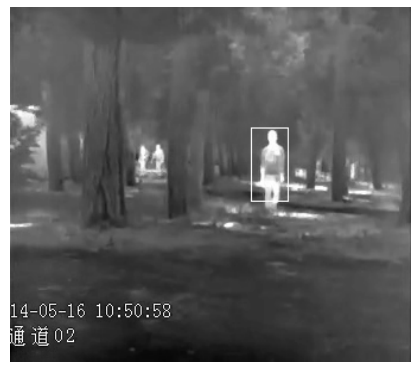

the 148th frame

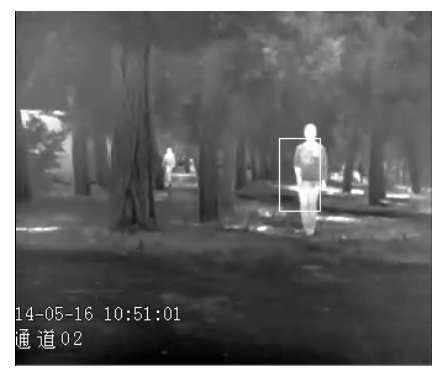

the 232th frame

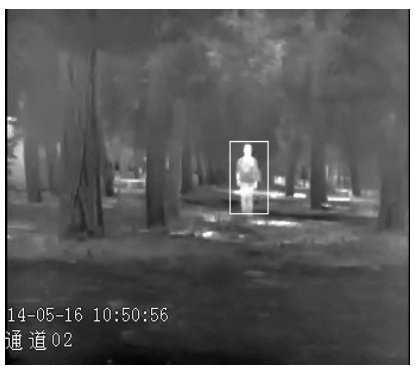

the 88th frame

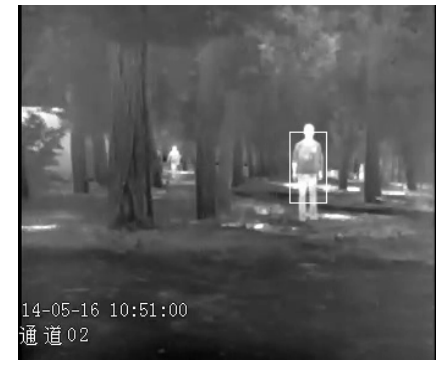

the 192th frame

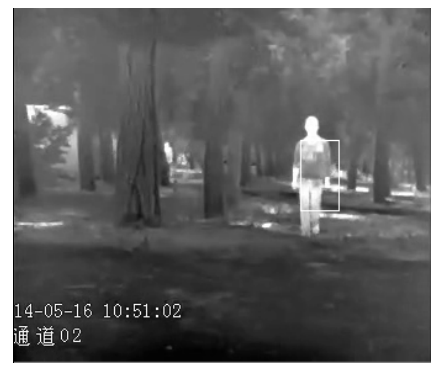

the 260th frame
Group A: classical Mean Shift algorithm

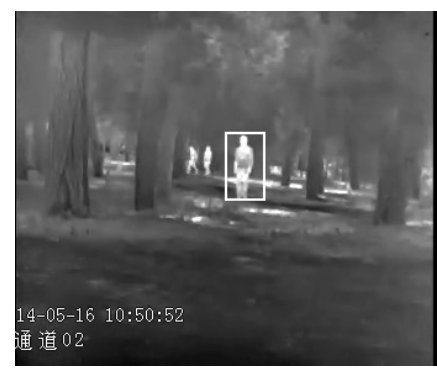

the 1st frame

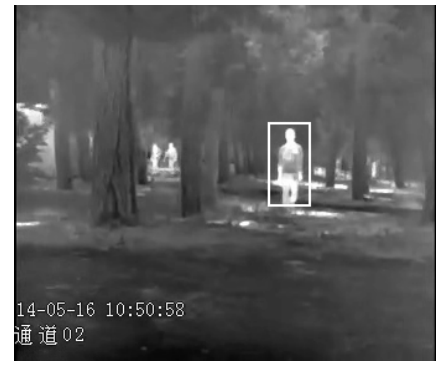

the 148th frame

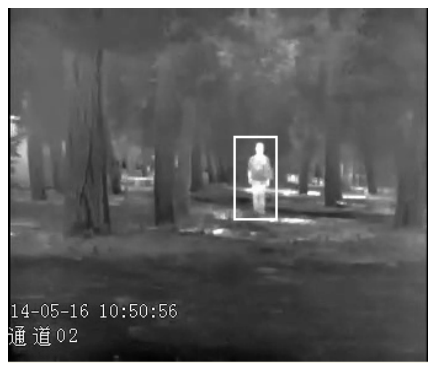

the 88th fram

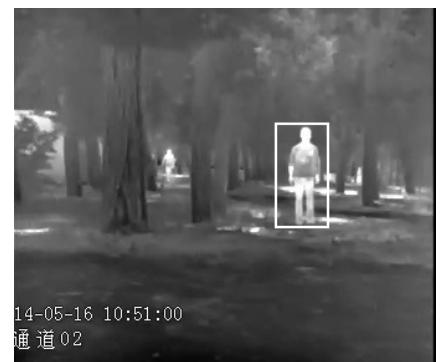

the 192th frame 


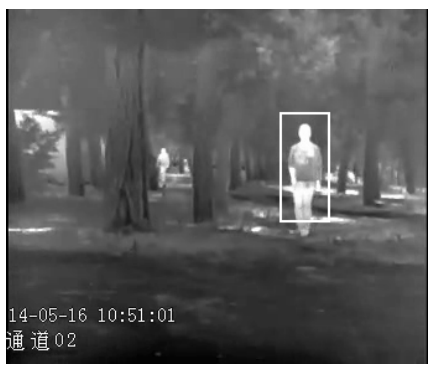

the 232th frame

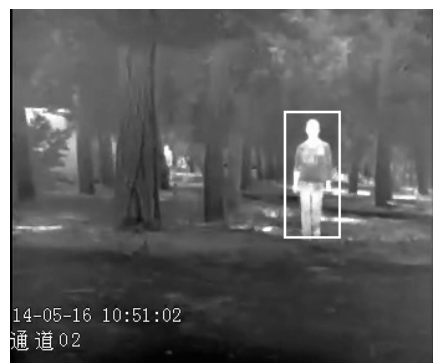

the 260th frame
Group B: The improved mean shift algorithm

Fig.2. Comparative results of classical Mean Shift algorithm and the improved algorithm.

It can be seen from Fig. 2 that the human body, a non-rigid target, has complex posture change and strong moving independence, so the classic mean shift used in group A can't automatically self-adapt the kernel window width algorithm to change the posture with the gradual largening of the tracking target with according to the target size. However, the tracking region area of image sequence can't change, which will cause the tracking region diverge from the tracking target gradually and the tracking size beyond the tracking region. It can be seen from group B that the tracking region could automatically adapt with the change of the target when the human body target had some posture change as the kernel window width could update according to the size of the human target after modifying the algorithm. Therefore, the tracking region didn't diverge from the target during the whole tracking and the tracking effect was better.

\section{CONCLUSION}

The modification method of the target modeling was introduce aiming at the disadvantages of the conventional mean shift. In the condition of background complexity and unobvious target feature of the infrared target image, the grayscale likelihood ratio weighted kernel function could suppress the interruption of background and make the pixel feature more obvious. Moreover, the representation model of the infrared target could also show the target feature of the infrared image sequence in the luminance to distance space. The modification of the classical mean shift makes the kernel window width of the tracking target self-adapt the change of the target size and simple posture, which solves the problem that the conventional mean shift can't track the change of the target size.

\section{REFERENCES}

[1] Pei-hua li. An improved Mean Shift tracking algorithm [J]. Journal of automation, 2007 (4) , pp.347-354.

[2] Bo Zhang,Weifeng Tian,Zhihua Jin .Joint tracking algorithm using particle filter and mean shift with target model updating[J]. Chinese optics letters (English edition), 2006,4(10), pp.569-572.

[3] Jing Qingfeng,Guo Qing.Adaptive compensating method for Doppler frequency shift using LMS and phase estimation[J]. Journal of systems engineering and electronics technology (English edition), 2009,20(5) , pp.913-919.

[4] Guo-wang gao, shang-qian liu, han-lin qin, etc. Under the strong background noise robustness of infrared target tracking algorithm [J]. Journal of xi 'an university of electronic science and technology (natural science edition), 2010, 5 (6) , pp.1098-1102.

[5] Sheng-li zhu, shan-an zhu. Kernel function adaptive bandwidth Mean shift tracking algorithm [J]. Journal of electrical engineering, 2006 (8), pp.11-16. 